\title{
Variaciones en la condición nutricional de langostas Panulirus argus (Decapoda: Palinuridae) en la región este del Golfo de Batabanó, Cuba
}

\author{
Alexander Lopeztegui Castillo, Norberto Capetillo Piñar \& Abel Betanzos Vega \\ Centro de Investigaciones Pesqueras, 5ta ave. y 246, Santa Fe, Playa, La Habana, Cuba; sasha@cip.telemar.cu, nor- \\ berto@cip.telemar.cu, abetanzos@cip.telemar.cu
}

Recibido 14-XII-2010. Corregido 20-V-2011. Aceptado 29-VI-2011.

\begin{abstract}
Variations of the nutritional condition of lobsters Panulirus argus (Decapoda: Palinuridae) in Eastern region of the Gulf of Batabanó, Cuba. Nutritional condition can affect survival and growth rate of crustaceans, and this is mostly affected by habitat conditions. This study describes the space-temporary nutritional changes in this commercially important species. With this aim, the variations in the nutritional condition (K) of lobsters from four zones (1,2,4 and 5) in the Gulf of Batabanó, Cuba, were determined. For this, the weight/length ratio $(\mathrm{K}=\mathrm{Pt} / \mathrm{Lt})$ was calculated using animals captured in 1981 and 2010. The nutritional condition between areas and sexes, and years and sexes, was contrasted by a bifactorial ANOVA, and the overall length and weight of lobsters were compared using a t-Test for independent samples and unifactorial ANOVA. It was found that the nutritional condition was significantly greater in males than in females. In addition, significant variations between zones were detected for both years. Nutritional condition of lobsters from Zone five was the highest for 1981, while it was Zone two for 2010. Lobsters nutritional state showed significant variations between years, being greater in $1981(2.34 \pm 0.84 \mathrm{~g} / \mathrm{mm})$ than in $2010(1.96 \pm 0.49 \mathrm{~g} / \mathrm{mm})$. The inter-zones variations as well as the inter-annual ones seem to be related to the reported variations of the bottom type and the vegetation cover. Seasonal variations in the abundance and distribution of benthic organisms, that constitute food for lobsters, could also be influencing. The differences between sexes, however, were assumed as a consequence of the methodology used and the sexual dimorphism of the species. Due to other K estimation methods, that do not include morphometric measurements, these differences were not detected. We suggested that the P. argus nutritional condition is a good estimator of the habitat condition. Besides, according to the applied $\mathrm{K}$ estimation methodology, it was found that different groups of lobsters that have resemblant nutritional condition, did not necessarily observe similarities in the overall mean length or weight, so they could exist under different habitat conditions. Rev. Biol. Trop. 60 (1): 263-271. Epub 2012 March 01.
\end{abstract}

Key words: Panulirus argus, nutritional condition, Gulf of Batabanó.

La langosta Panulirus argus (Latreille 1804), principal recurso pesquero de Cuba, es un organismo cuyas fases bentónicas (postlarvales) transcurren en gran medida asociadas a pastos marinos, donde encuentran mayormente alimento, y a arrecifes coralinos, donde encuentran elevada disponibilidad de refugio. Durante las noches, las langostas realizan movimientos de forrajeo (búsqueda de alimento) y atrapan principalmente moluscos (en su mayoría gastrópodos y bivalvos), crustáceos de menor tamaño, equinodermos y poliquetos (Herrera et al. 1991, Herrera \& Ibarzábal 1995); los que son integrantes de las comunidades zoobentónicas. Estos movimientos de forrajeo tienen lugar fundamentalmente en los pastos marinos, que cubren alrededor del $64.85 \%\left(13818 \mathrm{~km}^{2}\right)$ del área total sumergida $\left(21305 \mathrm{~km}^{2}\right)$ del Golfo de Batabanó, Cuba (Cerdeira-Estrada et al. 2008).

El estado o condición nutricional de las langostas es una medida del grado en que éstas han acumulado las reservas energéticas necesarias para la adecuada realización de las funciones fisiológicas y el crecimiento (Moore 
et al. 2000). Esta condición depende fundamentalmente de la disponibilidad de alimento y de la calidad del mismo (Lozano-Álvarez \& Aramoni-Serrano 1996). Además de esto, la disponibilidad de refugios y la ocurrencia de enfermedades pueden también influir en el estado nutricional de estos crustáceos (Pollock et al. 1997, McGarvey et al. 1999, Behringer \& Butler 2006), sin embargo, los cambios más evidentes en dicho estado son causados por períodos de hambruna relativamente prolongados.

Por esta razón, la condición nutricional de las langostas puede ser el resultado de la influencia de cualquier factor (o factores) que directa o indirectamente introduzca variaciones significativas en las comunidades bentónicas. Según Oliver \& MacDiarmid (2001), en especies que viven en hábitats de vegetación variada, y/o en las que los individuos cambian de hábitat en función de la fase del desarrollo ontogenético, como es el caso de $P$. argus, la condición nutricional de los organismos puede ser afectada por variaciones en el estado de la vegetación y los flujos de energía en el ecosistema.

El estado nutricional de las langostas puede estimarse mediante varios métodos uno de los cuales se basa en la relación entre el peso y el largo total de los individuos (Robertson et al. 2000, Briones-Fourzán et al. 2009). Aunque varios autores plantean que el índice basado en la relación peso/largo no responde tan rápidamente como otros a los cambios en las condiciones ambientales y a los períodos de hambruna (Dall 1974, Behringer \& Butler 2006); esta metodología presenta la ventaja de ser rápida, no destructiva, y de no requerir un elevado desarrollo tecnológico para su aplicación. Además, se ha planteado que en estadíos juveniles de especies de langosta como Jasus edwarsii, la relación peso/largo no es afectada por las variaciones fisiológicas que ocurren durante el proceso de muda (Oliver \& MacDiarmid 2001). Independientemente de la vía que se utilice para su cálculo, el estado nutricional de un grupo o población de langostas es consecuencia de la interacción de varios de los factores que condicionan su hábitat, por lo que las variaciones en dicho estado pueden ser indicativas de variaciones en la calidad del hábitat, las que pudieran implicar afectaciones a otros organismos del área.

En Cuba, la región del Golfo de Batabanó es la más importante zona de pesca de langosta debido a que de ella se extrae anualmente alrededor del 70\% de la captura nacional (Cruz et al. 2001, Puga 2005). Este estudio persigue como objetivos estimar la condición nutricional de langostas $P$. argus en la región este del Golfo de Batabanó, y determinar variaciones en dicha condición basado en comparaciones espacio-temporales.

\section{MATERIALES Y MÉTODOS}

El Golfo de Batabanó (GB) se ubica en la plataforma Suroccidental de Cuba, la más extensa del país (Cruz et al. 1990). Las langostas muestreadas fueron seleccionadas al azar de las capturas comerciales, razón por la cual fueron en su mayoría individuos mayores de $69 \mathrm{~mm}$ de largo de cefalotórax y en período de intermuda. Los ejemplares medidos provinieron de la región este del GB, de cuatro de las zonas de pesca pertenecientes a la empresa PESCAHABANA del municipio de Batabanó (Fig. 1).

En 1981 se trabajó con datos de 816 ejemplares (408 machos y 408 hembras) muestreados en los meses de septiembre-noviembre. En este caso se contabilizaron datos de 204 ejemplares por zona, estos datos fueron recopilados de las estadísticas del Centro de Investigaciones Pesqueras. En 2010, se midieron 35 ejemplares por zona, por lo cual se registraron datos de 140 ejemplares (62 machos y 78 hembras) medidos in situ entre los meses de junio-agosto.

Las mediciones de largo total (Lt) se realizaron con una cinta métrica $( \pm 1 \mathrm{~mm})$. Como medida de Lt se registró el largo total antenular, medido ventralmente desde el punto medio en la base entre las dos antenas hasta el punto medio posterior del telson. El peso total $(\mathrm{Pt}) \mathrm{de}$ los ejemplares se midió utilizando una balanza analítica $( \pm 1 \mathrm{~g})$. La condición nutricional $(\mathrm{K})$ de 


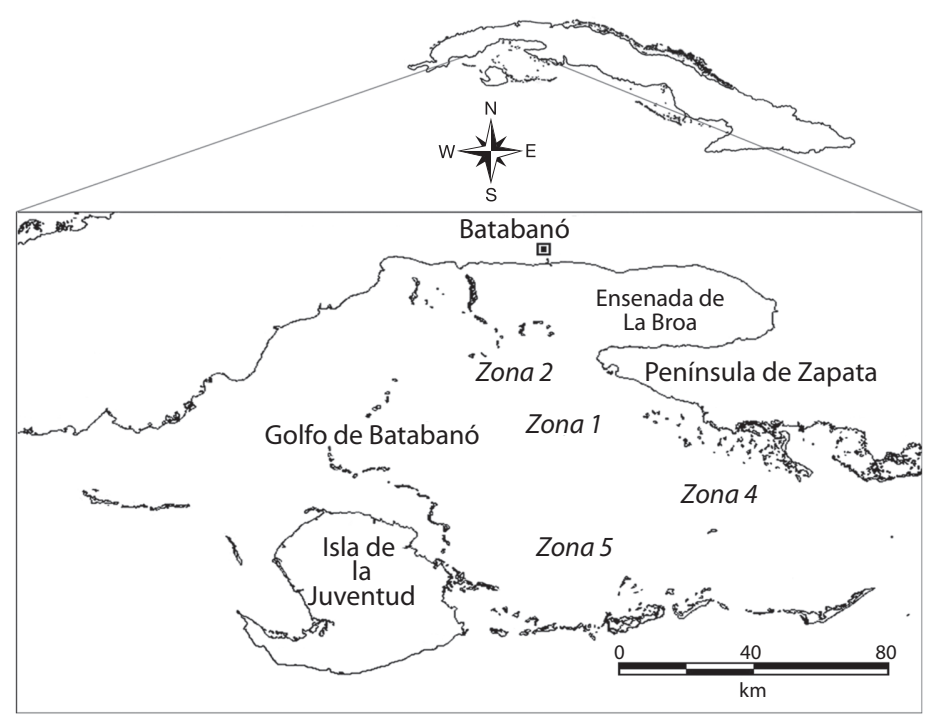

Fig. 1. Ubicación geográfica de las zonas de estudio en la región este del Golfo de Batabanó.

Fig. 1. Geographic location of study zones in Eastern region of the Gulf of Batabanó.

las langostas, se estimó mediante la relación Pt/ $\mathrm{Lt}$, la cual quedó expresada en $\mathrm{g} / \mathrm{mm}$.

Las comparaciones de $\mathrm{K}$ tanto entre zonas $(1,2,4$ y 5) y sexos como entre años (1981 y 2010), se realizaron mediante un análisis de varianza (ANOVA) bifactorial, en el que se tomó en cuenta además la interacción entre las variables según cada caso. Para comparar entre años el peso total y el largo total de los ejemplares, se aplicó una Prueba t para muestras independientes. Los intervalos de confianza fueron calculados en todos los casos para el $95 \%(<0.05)$. Para el procesamiento de los datos se utilizó Statistica 6.0 (StatSoft 2001).

\section{RESULTADOS}

La condición nutricional de las langostas presentó variaciones significativas entre zonas en ambos años analizados (1981: F[3, $808]=161.86, p<0.05 ; 2010: F[3,132]=2.7514$, $\mathrm{p}<0.05)$. En 1981 las langostas provenientes de la Zona cinco presentaron la mayor condición nutricional mientras que las de la Zona uno el estado nutricional más deficiente. Solamente las langostas provenientes de las Zonas uno y dos no presentaron diferencias significativas en su estado nutricional (Fig. 2A). En el año 2010 la mayor condición nutricional la presentaron las langostas provenientes de la Zona dos, y no se detectaron diferencias significativas en el estado nutricional de las langostas provenientes de las restantes zonas (Fig. 2B).

Además, se detectaron diferencias significativas entre sexos tanto en $1981(\mathrm{~F}=52.197$, $\mathrm{p}<0.05)$ como en $2010(\mathrm{~F}=13.068, \mathrm{p}<0.05)$. En ambos casos la condición nutricional de los machos fue mayor que la de las hembras (Fig. 3 A y B).

Sin embargo, en lo que respecta a la interacción entre los factores zona y sexo, se encontró diferencias en la influencia para cada año. En 1981, la interacción zona-sexo influye significativamente $(\mathrm{F}=72.630, \mathrm{p}<0.05)$ sobre la condición nutricional de las langostas (Fig. 4A). Para el año 2010 en cambio (Fig. 4B), la influencia de esta interacción no fue significativa $(\mathrm{F}=1.2073, \mathrm{p}>0.05)$.

La condición nutricional de las langostas en 1981 fue de $2.34 \pm 0.84 \mathrm{~g} / \mathrm{mm}$. En el año 

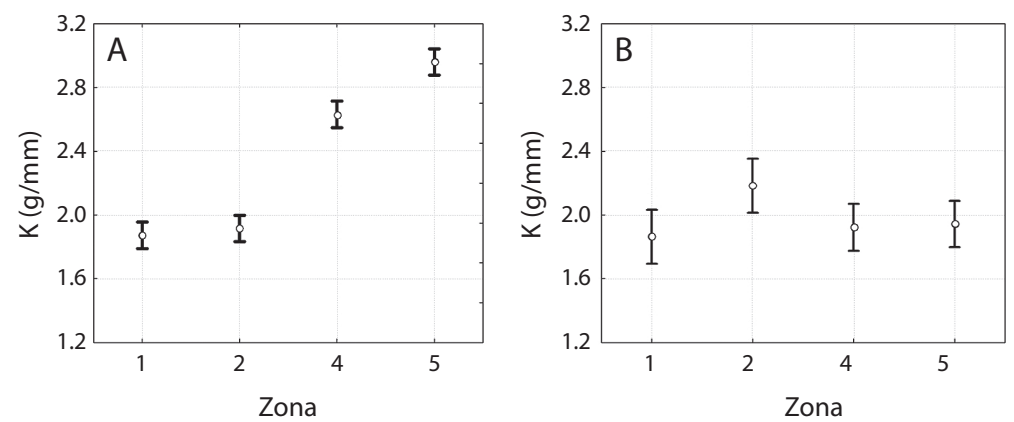

Fig. 2. Comparación por zonas de la condición nutricional (K) de langostas P. argus de la región este del Golfo de Batabanó. A: año 1981, B: año 2010.

Fig. 2. Comparison by zones of the nutritional condition (K) of lobsters P. argus in Eastern region of the Gulf of Batabanó. A: year 1981, B: year 2010 .
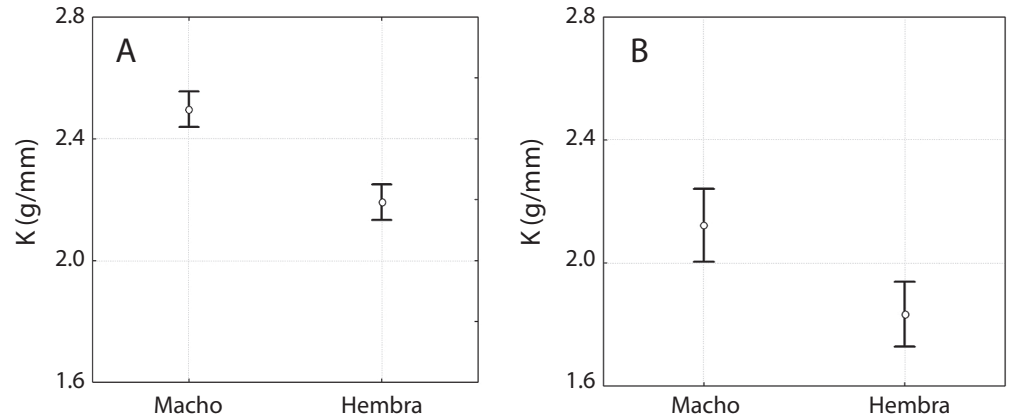

Fig. 3. Comparación por sexos de la condición nutricional (K) de langostas P. argus de la región este del Golfo de Batabanó. A: año 1981, B: año 2010.

Fig. 3. Comparison by sexes of the nutritional condition (K) of lobsters P. argus in Eastern region of the Gulf of Batabanó. A: year 1981, B: year 2010 .
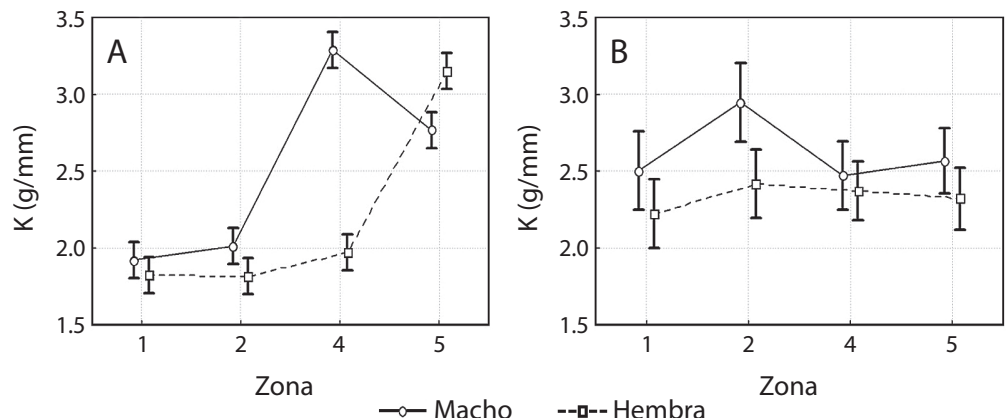

Fig. 4. Influencia de la interacción entre los factores zona y sexo sobre la condición nutricional (K) de langostas P. argus de la región este del Golfo de Batabanó. A: año 1981, B: año 2010.

Fig. 4. Influence of interaction between the factors zone and sex on the nutritional condition (K) of lobsters $P$. argus in Eastern region of the Gulf of Batabanó. A: year 1981, B: year 2010. 
2010 esta condición fue de $1.96 \pm 0.49 \mathrm{~g} / \mathrm{mm}$. Como se muestra en la Figura 5A, existen diferencias entre años, la condición nutricional de los individuos fue significativamente mayor en 1981 ( $\mathrm{F}=26.900, \mathrm{p}<0.05)$. Las diferencias significativas entre sexos $(\mathrm{F}=16.122, \mathrm{p}<0.05)$ se mantienen al tener en cuenta los individuos de ambos años agrupados (Fig. 5B).
La comparación mediante una Prueba $t$ para muestras independientes permitió detectar que existen diferencias entre años tanto en el largo total de los ejemplares como en el peso de los mismos. En ambos casos los valores obtenidos son significativamente menores en el 2010, sin embargo, la disminución en cuanto al peso es de mayor magnitud (Cuadro 1).
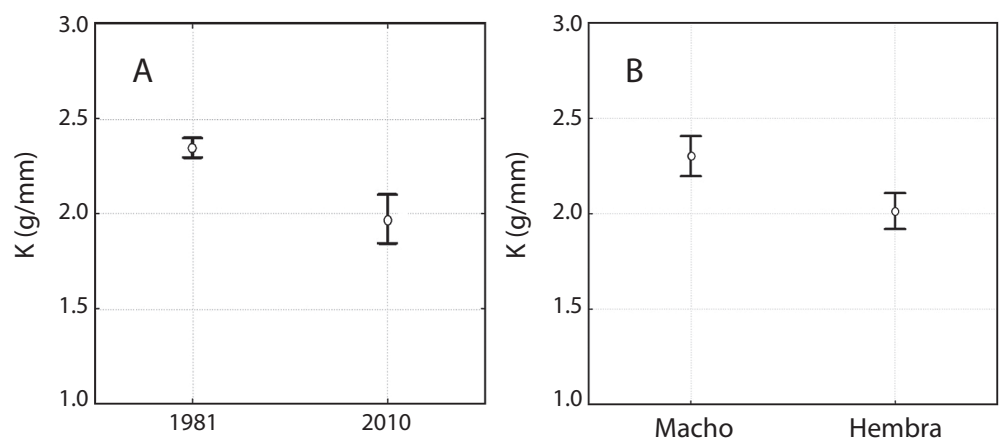

Fig. 5. Comparación interanual (A) e inter-sexos (B) de la condición nutricional (K) de langostas P. argus residentes en la porción este del Golfo de Batabanó.

Fig. 5. Inter-annual (A) and intersexes (B) comparison of the nutritional condition (K) of lobsters P. argus resident in Eastern region of the Gulf of Batabanó.

\section{CUADRO 1}

Comparación interanual del largo total (Lt) y el peso (P) de langostas $P$. argus provenientes de la región este del Golfo de Batabanó

TABLE 1

Inter-annual comparison of the overall length (Lt) and weight $(\mathrm{Pt})$ of lobsters $P$. argus captured in Eastern region of the Gulf of Batabanó

\begin{tabular}{|c|c|c|c|c|c|c|c|}
\hline & Media & $\mathrm{t}$ & g.1. & $\mathrm{N}$ & D.E. & $\mathrm{F}$ & $\mathrm{p}$ \\
\hline Lt (mm) 1981 & 260.31 & \multirow{2}{*}{2.1964} & \multirow{2}{*}{954} & 816 & 42.2059 & \multirow{2}{*}{2.4112} & \multirow{2}{*}{$0.02830^{*}$} \\
\hline \multirow[t]{2}{*}{ Lt (mm) 2010} & 252.20 & & & 140 & 27.1808 & & \\
\hline & Media & $\mathrm{t}$ & g.1. & $\mathrm{N}$ & D.E. & $\mathrm{F}$ & $\mathrm{p}$ \\
\hline Pt (g) 1981 & 644.39 & \multirow{2}{*}{4.5699} & \multirow{2}{*}{954} & 816 & 352.7689 & \multirow{2}{*}{3.6678} & \multirow{2}{*}{$0.00000^{*}$} \\
\hline Pt (g) 2010 & 504.94 & & & 140 & 184.1978 & & \\
\hline
\end{tabular}

\section{DISCUSIÓN}

Las diferencias entre zonas, e incluso entre años, que presenta la condición nutricional de las langostas, podrían estar relacionadas con la disponibilidad de alimento, la cual puede variar en dependencia de múltiples factores. Uno de éstos es el buen desarrollo de los pastos marinos, que propicia el establecimiento de las comunidades bentónicas que constituyen la fuente de alimento para las langostas. Otro factor que puede determinar la comunidad 
bentónica establecida en una zona dada, y puede por tanto influenciar la disponibilidad de alimento para las langostas, es el tipo de fondo según el tamaño de los granos del sedimento (Gómez et al. 1980). Diversas investigaciones realizadas en el Golfo de Batabanó durante el período estudiado (1981-2010), demuestran que estos factores han variado visiblemente.

Para el primer quinquenio de la década 1980`s, la cobertura vegetal (Thalassia testudimun Banks ex König y macroalgas) en la porción este del GB fue descrita como vegetación de densidad media (Jiménez \& Alcolado 1990). Páez et al. (1991) reportaron para el segundo quinquenio de esta década una variación en dirección Norte-Sur que va desde fondo fangoso en las cercanías de la Península de Zapata y la Ensenada de la Broa (Zonas uno y dos), hasta fondo areno-fangoso en la porción más al Sur (Zona cinco). Hacia el centro y en la porción más al este (Zona cuatro), los fondos fueron reportados en su mayoría como arenosos con alguna vegetación. Estas condiciones pueden ser la causa de que en 1981 las langostas presentaran un mejor estado nutricional, debido a que propician el buen desarrollo de las comunidades bentónicas. La biomasa de organismos bentónicos, fundamentalmente de moluscos y crustáceos, se estimó entre media y alta para el período 1981-1985 (Alcolado 1990). Según Gómez et al. (1980), el biotopo arenoso presenta las menores densidades de organismos del bentos, de manera que el sustrato fangoso con vegetación más adecuado para el establecimiento de los mismos.

En la actualidad, los fondos en el área estudiada presentan en esencia poca variación y están cubiertos por pastos marinos de baja densidad, con pequeños parches de pastos de densidad entre media y alta (Cerdeira-Estrada et al. 2008). Espacialmente, esta situación podría fundamentar la poca variación entre la condición nutricional de las langostas de las diferentes zonas en el año 2010. Desde un punto de vista temporal, las variaciones en cuanto al tipo de fondo y su cobertura vegetal, podrían en parte explicar que para este año 2010 las langostas presentaran una condición nutricional significativamente menor que en 1981. Ya para los primeros años del siglo XXI se reporta para el GB una vegetación escasa y parcheada (Martínez-Daranas et al. 2005). La disminución que entre 1985 y 2005 experimentó la cobertura de fanerógamas marinas ha sido estimada en 24.9\% (Areces et al. 2006). En 2003-2004, con respecto a 1981-1985, Arias-Schreiber et al. (2008) reportan una disminución significativa del bentos, fundamentalmente las comunidades de esponjas, equinodermos, poliquetos $\mathrm{y}$ en menor medida de moluscos.

Las variaciones señaladas anteriormente en cuanto a distribución y densidad de pastos marinos, y al estado de las comunidades bentónicas, pueden estar relacionadas con actividades antrópicas como el represamiento de los ríos, y factores climáticos como la incidencia de huracanes y tormentas tropicales. Entre otros efectos, el represamiento limita la cantidad de nutrientes que ingresa al mar producto del escurrimiento (Baisre 2006). Los ciclones tropicales provocan afectaciones locales cuya permanencia depende de la intensidad, frecuencia y duración de estos eventos. Según Puga et al. 2009, la capacidad de embalse en el GB comenzó a incrementarse desde 1971, fue mayor que el promedio en 1980, y alcanzó su máximo valor en 1991. Estos mismos autores reportaron que la intensidad y frecuencia de huracanes que han afectado el área entre 1996 y 2005, fue mayor que en años anteriores.

Las interacciones entre los diferentes factores (año, sexo o zona) y su influencia sobre la condición nutricional, también sugieren que las variaciones en dicha condición están relacionadas con las variaciones de hábitat. En 1981, la relación zona-sexo afectó significativamente el estado nutricional de los ejemplares. Es decir, una parte significativa de la variación de $\mathrm{K}$ puede ser explicada por la relación existente entre los factores zona y sexo (Fig. 4). Para este año, el GB presentaba no sólo mejor condición general de los fondos sino una mayor variedad en cuanto a tipo de fondo, cobertura vegetal y organismos bentónicos asociados. En 2010 sin embargo, los fondos presentan una condición menos heterogénea y más deteriorada, lo que 
se refleja en la influencia no significativa de la relación (interacción) zona-sexo sobre el estado nutricional de las langostas. Es decir, para este año 2010 la relación zona-sexo sustenta muy poca o ninguna variación en la condición nutricional.

La disponibilidad de alimento para las langostas está también determinada por las variaciones estacionales de las comunidades bentónicas, de manera que esta es otra de las razones que puede determinar variaciones temporales en la condición nutricional (LozanoÁlvarez \& Aramoni-Serrano 1996). Debido a que los muestreos en 1981 se realizaron en septiembre y noviembre, y los correspondientes a 2010 se realizaron entre junio y agosto, parte de las diferencias entre estos años con respecto a la condición nutricional podrían deberse a tales variaciones estacionales. Sin embargo, la influencia de la estacionalidad del bentos en las diferencias de $\mathrm{K}$ entre años se considera de menor importancia que los efectos de este estudio, puesto que la condición nutricional resultó menor en 2010, año en que los meses de muestreo coinciden con los de mayor proliferación de organismos en las comunidades bentónicas (Lozano-Álvarez \& Aramoni-Serrano 1996), razón por la cual debería ser mayor la disponibilidad de alimento y por tanto la condición nutricional de los ejemplares. Por otra parte, la estacionalidad de las comunidades bénticas en el GB ha sido muy poco estudiada, por lo que se recomienda la realización de estudios al respecto.

Las diferencias entre sexos, sin embargo, podrían deberse a particularidades morfológicas propias de la especie. Otras metodologías para la determinación del estado nutricional de las langostas, basadas no en mediciones morfométricas sino en parámetros fisiológicos como el índice de refracción de proteínas en la hemolinfa, o la composición y peso del hepatopáncreas, no detectan estas diferencias (Oliver \& MacDiarmid 2001, Briones-Fourzán et al. 2003, Briones-Fourzán et al. 2009). Como característica natural en $P$. argus, la proporción largo del cefalotórax/largo abdominal es ligeramente mayor en machos que en hembras. Esta expresión de dimorfismo sexual puede ser la causante de que la condición nutricional estimada mediante una relación peso-largo, muestre diferencias significativas entre los sexos.

La deficiencia nutricional de un individuo dentro de una población puede deberse a razones particulares que pueden no tener relación con el ambiente, sin embargo, un estado nutricional no satisfactorio como condición media en una población, está muy probablemente ligado a causas ambientales generalizadas (Oliver \& MacDiarmid (2001), como las descritas anteriormente. Así, tal como el estado de las comunidades bentónicas se considera exponente del potencial biótico y la calidad de los fondos en un sitio determinado (Alcolado 1999), la abundancia y distribución de langostas, unido a la condición nutricional de las mismas, podría considerarse reflejo de las condiciones generales del hábitat en que se encuentren. Según los resultados obtenidos, se puede plantear la existencia de una relación cualitativa directa entre el deterioro de los fondos (pérdida de vegetación submarina y fauna asociada) y la disminución de la condición nutricional de langostas $P$. argus de la región este del Golfo de Batabanó.

Aunque se comprobó que existe una disminución significativa de la longitud total y el peso total de las langostas en 2010 con respecto a 1981 (Tabla 1), no es sólo esta disminución la que fundamenta que también para este año la condición nutricional sea significativamente menor. Dado que K se estimó mediante la relación $\mathrm{Pt} / \mathrm{Lt}$, si ambas medidas disminuyen en la misma proporción no se afectará la condición nutricional resultante. Para que sea afectada (disminuida), es preciso que, como en este estudio, la disminución en el peso de los ejemplares sea mayor que la disminución en la talla de los mismos. Referente al año 1981, en el 2010 la longitud total media de las langostas disminuyó en $8.11 \mathrm{~mm}$, lo que significa el $3.12 \%$ de la longitud total media de las langostas medidas en 1981. El peso medio en cambio presenta una disminución de $139.45 \mathrm{~g}$, lo que significa el $21.64 \%$ del peso medio de las langostas pesadas en 1981 . 
Estos hechos sirven como base para afirmar que las variaciones de $\mathrm{K}$ son siempre consecuencia de variaciones en el peso y/o la talla de los ejemplares, lo que podría ocurrir como respuesta a variaciones ambientales; sin embargo, lo contrario no siempre se cumple. Cuando existen cambios en la talla y el peso de las langostas, no siempre estos cambios se reflejan en el resultado de la relación $\mathrm{Pt} / \mathrm{Lt}$, que estima la condición nutricional (es el caso en que el peso y la talla aumentan o disminuyen en la misma proporción). Consecuentemente, si se tienen dos grupos de langostas con similares valores medios de $\mathrm{K}$, no puede asegurarse que no existen diferencias entre ambos grupos hasta tanto no se compruebe que los valores medios de peso y longitud total (talla) son igualmente similares.

\section{AGRADECIMIENTOS}

A las empresas pesqueras de Batabanó e Isla de la Juventud por su colaboración durante la realización de los muestreos y por el apoyo logístico brindado a la salida de cada crucero de investigación. Al investigador Gerardo Suárez por sus recomendaciones metodológicas y sus aportes bibliográficos.

\section{RESUMEN}

En Cuba, la región del Golfo de Batabanó es la más importante zona de pesca de langosta debido a que de ella se extrae anualmente alrededor del $70 \%$ de la captura nacional. Con el objetivo de determinar variaciones espacio-temporales en la condición nutricional (K) de langostas Panulirus argus del Golfo de Batabanó, Cuba, se calculó dicha condición mediante una relación pesolargo $(\mathrm{K}=\mathrm{Pt} / \mathrm{Lt})$, para lo que se utilizaron ejemplares de cuatro zonas (uno, dos, cuatro y cinco) del este del golfo, capturados en los años 1981 y 2010. Se encontró que en todos los casos analizados la condición nutricional fue significativamente mayor en machos que en hembras. Se detectaron variaciones significativas entre zonas en 1981 y 2010. También entre años el estado nutricional de las langostas presenta variaciones significativas, de manera que en $1981(2.34 \pm 0.84 \mathrm{~g} / \mathrm{mm})$ fue mayor que en 2010 $(1.96 \pm 0.49 \mathrm{~g} / \mathrm{mm})$. Tanto las variaciones interzonales como las interanuales parecen estar relacionadas con variaciones reportadas en el tipo de fondo y la cobertura vegetal. Variaciones estacionales en la abundancia y distribución de organismos bentónicos que constituyen alimento para las langostas también podrían estar influyendo. Las diferencias entre sexos en cambio, se suponen consecuencia de la metodología utilizada y del dimorfismo sexual característico de esta especie, por cuanto otros métodos de estimación de $\mathrm{K}$ que no involucran mediciones morfométricas, no detectan estas diferencias.

Palabras clave: Panulirus argus, condición nutricional, Golfo de Batabanó.

\section{REFERENCIAS}

Alcolado, P.M. 1990. El Bentos de la Macrolaguna del Golfo de Batabanó. Academia. La Habana, Cuba.

Alcolado, P.M. 1999. Monitoreo Biológico Marino, p.199222. In F. Martínez-Luzardo, L. Sorina-González, J. Torres-Hernández, L.A. Lima-Carzola, J.L. Borroto-Portela, J.R. Estévez-Álvarez, J.L. SantanaRomero, O. Arjona-Vázquez, M. Arcia-Rodríguez \& M. Roque-Molina (eds.). Contribución a la Educación y la Protección Ambiental. Academia, La Habana, Cuba.

Areces, A.J., A. García, C. Martínez, G. Hidalgo, S. Castellanos, K. Cantelar, J.C. Martínez, R. del Valle, M. Abreu, Z. Marcos \& D. Pérez. 2006. Hacia el uso sostenible del Golfo de Batabanó: análisis de sistemas y modelación de escenarios. Inf. Cient. Téc., IDO, La Habana, Cuba.

Arias-Schreiber, M., M. Wolff, M. Cano, B. MartínezDaranas, Z. Marcos, G. Hidalgo, S. Castellanos, R. del Valle, M. Abreu, J.C. Martínez, J. Diaz \& A. Areces. 2008. Changes in benthic assemblages of the Gulf of Batabanó (Cuba) results from cruises undertaken during 1981-85 and 2003-04. Pan-Am. J. Aquat. Sci. 3: 49-60.

Baisre, J.A. 2006. Assessment of nitrogen flows into the Cuban landscape. Biogeochemistry 79: 91-108.

Behringer, D.C. \& M.J. Butler. 2006. Density-dependent population dynamics in juvenile Panulirus argus (Latreille): the impact of artificial density enhancement. J. Exp. Mar. Biol. Ecol. 334: 84-95.

Briones-Fourzán, P., V. Castañeda-Fernández de Lara, E. Lozano-Álvarez \& J. Olivo-Estrada. 2003. Feeding ecology of the three juvenile phases of the spiny lobster Panulirus argus in a tropical reef lagoon. Mar. Biol. 142: 855-865.

Briones-Fourzán, P., K. Baeza-Martínez \& E. LozanoÁlvarez. 2009. Nutritional indices of juvenile Caribbean spiny lobsters in a Mexican reef lagoon: Are 
changes over a 10-year span related to the emergence of Panulirus argus Virus 1 (PaV1)? J. Exp. Mar. Biol. Ecol. 370: 82-88.

Cerdeira-Estrada, S., S. Lorenzo-Sánchez, A. Areces-Mallea \& C. Martínez-Bayón. 2008. Cartografía de la distribución espacial de los hábitats bentónicos en el Golfo de Batabanó utilizando imágenes Landsat-7. Cienc. Mar. 34: 213-222.

Cruz, R., E. Díaz, M. Báez \& R. Adriano. 2001. Variability in recruitment of multiple life stages of the Caribbean spiny lobster, Panulirus argus, in the Gulf of Batabanó, Cuba. Mar. Freshwat. Res. 52: 1263-1270.

Cruz, R., J.A. Baisre, E. Díaz, R. Brito, W. Blanco, C. García \& C. Carrodeguas. 1990. Atlas Biológico-Pesquero de la Langosta en el archipiélago Cubano. Pub. Esp. Rev. Cuba. Invest. Pesq. y Rev. Mar y Pesca.

Dall, W. 1974. Indices of nutriotional state in the western rock lobster Panulirus longipes (Milne Edwards). I. Blood and tissue constituents and water content. J. Exp. Mar. Biol. Ecol. 16: 167-180.

Gómez, O., D. Ibarzábal \& A. Silva. 1980. Evaluación cuantitativa del bentos en la región suroccidental de Cuba. Inf. Cient. Téc. IDO, Acad. Cienc. Cuba 149: $1-25$.

Herrera, A. \& D. Ibarzábal. 1995. Aspectos ecológicos de la langosta Panulirus argus en los arrecifes de la plataforma cubana. Rev. Cub. Invest. Pesq. 19: 59-63.

Herrera, A., D. Ibarzábal, J. Foyo, J. Espinosa, R. Brito, G. González, E. Díaz, G. Goteras \& C. Arrinda. 1991. Alimentación natural de la langosta $P$. argus en la región de Los Indios (Plataforma SW de Cuba) y su relación con el bentos. Rev. Invest. Mar. 12: 172-182.

Jiménez, D.C. \& P.M. Alcolado. 1990. Características del macrofitobentos de la macrolaguna del Golfo de Batabanó, p. 8-11. In P.M. Alcolado (ed.). El Bentos de la Macrolaguna del Golfo de Batabanó. Academia, La Habana, Cuba

Lozano-Álvarez, E. \& G. Aramoni-Serrano. 1996. Alimentación y estado nutricional de las langostas Panulirus inflatus y Panulirus gracilis (Decapoda: Palinuridae) en Guerrero, México. Rev. Biol. Trop. 44/45: 453-461.

Martínez-Daranas, B., M. Cano-Mallo, C. Martínez-Bayón, S. Lorenzo, D.M. Pérez-Zayas, M. Esquivel-Céspedes
\& M. Hernández-González. 2005. Estado de conservación de los pastos marinos del Golfo de Batabanó. Inf. Cient. Téc., IDO, La Habana, Cuba.

McGarvey, R., G.J. Ferguson \& J.H. Prescott. 1999. Spatial variation in mean growth rates at size of southern rock lobster, Jasus edwardsii, in South Australian waters. Mar. Freshwat. Res. 50: 333-342.

Moore, L.E., D.M. Smith \& N.R. Loneragan. 2000. Blood refractive index and whole body lipid content as indicators of nutricional condition for penaeid prawns (Decapada: Penaeidae). J. Exp. Mar. Biol. Ecol. 244: 131-143.

Oliver, M.D. \& A.B. MacDiarmid. 2001. Blood refractive index and ratio of weigth to carapace length as indices of nutricional condition in juvenile rock lobsters (Jasus edwarsii). Mar. Freshwat. Res. 52: 1395-4000.

Páez, J., N. Revilla \& J. Baisre. 1991. Relación entre la captura por unidad de área de la langosta (Panulirus argus) y los diferentes biotopos en el Golfo de Batabanó, Cuba. Rev. Invest. Mar. 12: 269-279.

Pollock, D.E., A.C. Cockcroft \& P.C. Goosen. 1997. A note on reduced rock lobster growth rates and related environmental anomalies in the southern Benguela, 1988-1995. S. Afr. J. Mar. Sci. 18: 287-293.

Puga, R. 2005. Modelación bioeconómica y análisis de riesgo de la pesquería de langosta espinosa Panulirus argus (Latreille, 1804) en el Golfo de Batabanó, Cuba. Tesis de Doctorado, Centro de Investigaciones Biológicas del Noroeste, La Paz, México.

Puga, R., R. Piñeiro, N. Capetillo, M.E. de León \& S. Cobas. 2009. Caso de estudio No. 2: Estado de la pesquería de la langosta espinosa (Panulirus argus) y su relación con factores ambientales y antrópicos en Cuba, p. 1-29. In A. Hernández (ed.). Evaluación de las posibles afectaciones del cambio climático a la biodiversidad marina y costera de Cuba. ISBN: 978959-298-017-4. (También disponible en línea: www. redciencia.cu).

Robertson, D.N., M.J. Butler \& F.C. Dobbs. 2000. An evaluation of lipid and morphometric based indices of nutriotional condition for early benthic stage of spiny lobsters Panulirus argus. Mar. Freshwat. Behav. Physiol. 33: 161-171.

StatSoft, Inc. 2001. STATISTICA (data analysis software system), version 6 . 
